\title{
SiC Multilayer Photonic Structures with Self Optical Bias Amplification
}

\author{
Manuela Vieira ${ }^{1,2,3}$, Manuel A. Vieira ${ }^{1,2}$, Paula Louro ${ }^{1,2}$, Alessandro Fantoni ${ }^{1,2}$, \\ Manuel Barata ${ }^{1,2}$, and Vitor Silva ${ }^{1,2}$ \\ ${ }^{1}$ Electronics Telecommunications and Computer Dept, ISEL, Lisbon, Portugal \\ ${ }^{2}$ CTS-UNINOVA, Quinta da Torre, 2829-516, Caparica, Portugal \\ ${ }^{3}$ DEE-FCT-UNL, Quinta da Torre, 2829-516, Caparica, Portugal
}

\begin{abstract}
Characteristics of tunable wavelength pi'n/pin filters based on a-SiC:H multilayered stacked cells are studied both experimental and theoretically. Results show that the device combines the demultiplexing operation with the simultaneous photodetection and self amplification of the signal. An algorithm to decode the multiplex signal is established. A capacitive active bandpass filter model is presented and supported by an electrical simulation of the state variable filter circuit. Experimental and simulated results show that the device acts as a state variable filter. It combines the properties of active highpass and low-pass filter sections into a capacitive active band-pass filter using a changing photo capacitance to control the power delivered to the load.
\end{abstract}

Keywords: A-SiC:H Pi'n/Pin Optical Filters, Self Amplification, Photodetection, Electrical Model, Optoelectronic Logic Programming, MUX/DEMUX Device.

\section{Introduction}

An optical filter comprises a material that has nonlinear, i.e., amplitude-dependent, response to each incident light wave. This nonlinearity provides the possibility for selectively removing or adding wavelengths and can compensate for coupling losses and be used to boost signal before demultiplexing.

In the WDM signal transmission, three channels (red, green and blue) are demultiplexed using wavelength selective channel filters. Background light can be changed to select different wavelength channels. By superimposing background illumination to the pulsed channel the device behaves as a filter, producing signal attenuation, or as an amplifier, producing signal gain, depending on the channel/background wavelength combination. This effect [1] can be described in terms of a wavelength controlled photo-capacitance.

Optical filters were produced and optimized for a fine tuning of a specific wavelength. Depending on the wavelength of the external background, the monolithic device acts either as a short- or a long- pass band filter or as a band-stop filter. 


\section{Contribution to Value Creation}

Systems that transmit, receive, and/or process intelligence require some form of modulation, which is the deliberate distortion of a carrier to impress intelligence (data) upon it that allows the subsequent recovery of the information. Data transmission can be improved using the well known wavelength division multiplexing-demultiplexing technique. The plastic optical fiber is a suitable, promising solution as transmission medium for short range communications. Besides it is more resilient to damage than glass due to its intrinsic material characteristics. It is easier to terminate, polish, and connect as well, which can reduce the cost of installation and maintenance. So, the conception of new devices based on new materials for signal (de)multiplexing in the visible spectrum is a demand in the direction of an increased potential for value creation. Such technological innovation can influence economic transformation, create value in the society and ultimately improve individuals' lives. Therefore, the theoretical approach presented in this research is useful in examining a particular aspect of value creation, configuring firm activities (automotive industry, aerospace, medical and housing sectors) to create value combining unique resources and capabilities, devices based on new materials for signal (de)multiplexing in the visible spectrum, in the direction of an increased potential for value creation.

\section{Device Configuration and Operation}

The tunable optical devices were produced by PECVD and optimized for a proper fine tuning of a specific wavelength. The active device consists of a $\mathrm{p}-\mathrm{i}^{\prime}(\mathrm{a}-\mathrm{SiC}: \mathrm{H})-\mathrm{n} / \mathrm{p}-$ $\mathrm{i}(\mathrm{a}-\mathrm{Si}: \mathrm{H})-\mathrm{n}$ heterostructure with low conductivity doped layers.

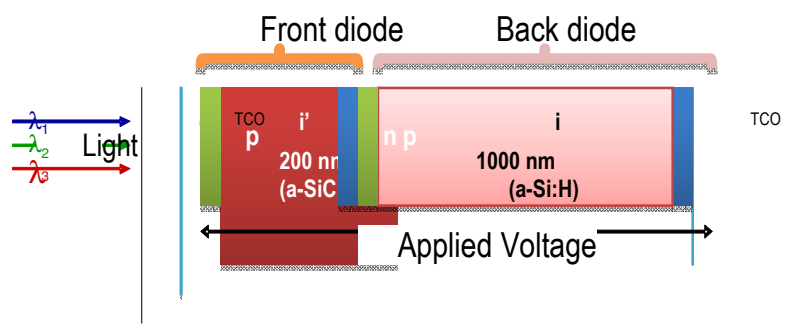

Fig. 1. Device configuration

The thicknesses and optical gap of the thin 1'- $(200 \mathrm{~nm} ; 2.1 \mathrm{eV})$ and thick i- $(1000 \mathrm{~nm} ; 1.8 \mathrm{eV})$ layers are optimized for light absorption in the blue and red ranges, respectively [2]. As a result, both front and back pin structures act as optical filters confining, respectively, the blue and the red optical carriers. Device configuration is depicted in Fig. 1.

The manipulation of the magnitude is achieved by changing the wavelength (color channels) of the modulated lights and its frequency. By providing a complex illumina- 
tion of the device combining radiations with different and complementary penetration depths it is possible to control the spectral response of the device.

In the WDM signal transmission, three channels (red, green and blue) are demultiplexed using wavelength selective channel filters. Background light can be changed to select different wavelength channels. By superimposing background illumination to the pulsed channel the device behaves as a filter, producing signal attenuation, or as an amplifier, producing signal gain, depending on the channel/background wavelength combination. This effect is reported and fully discussed elsewhere [1].

\section{Voltage Controlled Sensitivity}

To test the sensitivity of the device under different applied voltage and optical bias three modulated monochromatic lights channels: red (R: $626 \mathrm{~nm})$, green $(\mathrm{G}: 524 \mathrm{~nm})$ and blue (B: 470nm) and their polychromatic combinations (multiplexed signal) illuminated separately the device and the generated photocurrent was measured under positive and negative voltages, with and without steady state red, green and blue optical bias. The light modulation frequency of each channel was chosen to be multiple of the others to ensure a synchronous relation of ON-OFF states along each cycle and the optical powers were adjusted to give different signal magnitudes at $-8 \mathrm{~V}$.

Fig. 2 displays the single (a) and the multiplexed (b) signals under negative (-8V) and positive $(+1 \mathrm{~V})$ electrical bias.

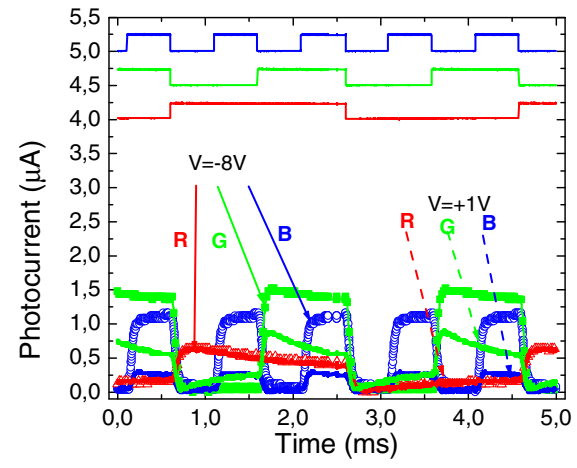

(a)

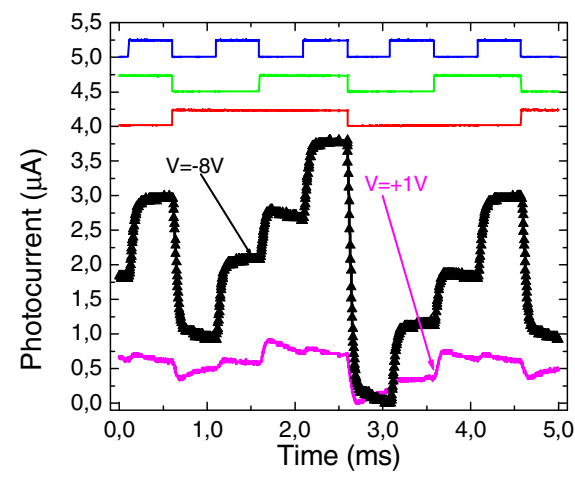

(b)

Fig. 2. Single (R, G and B) (a) and combined (b) signals under $-8 \mathrm{~V}$ (symbols) and $+1 \mathrm{~V}$ (lines) applied voltage

Results show that the red input signal remains constant, the green decreases and the blue almost disappears as the voltage changes from negative to positive. The output multiplexed signal, obtained with the combination of the three optical sources, depends on both the applied voltage and on the ON-OFF state of each input optical channel. Under negative bias, there are eight separate levels $\left(2^{3}\right.$ level encoding) while under positive bias they were reduced to one half $\left(2^{2}\right.$ level encoding). The highest 
level appears when all the channels are ON and the lowest if they are OFF. Furthermore, the levels ascribed to the mixture of three or two input channels are higher than the ones due to the presence of only one (R, G, B).

\section{$5 \quad$ Optical Bias Controlled Sensitivity}

In Fig. 3, at $-8 \mathrm{~V}$, the same red, green and blue monochromatic input channels (a) and their polychromatic combination but with different bit sequences (b), without $(\varphi=0)$ and with red steady state optical bias $(624 \mathrm{~nm}, \varphi=73 \mathrm{mWcm}-2)$ are displayed at a bit rate of $6000 \mathrm{bps}$. The bit sequences to drive the channels are shown in the top of the figure to guide the eyes.

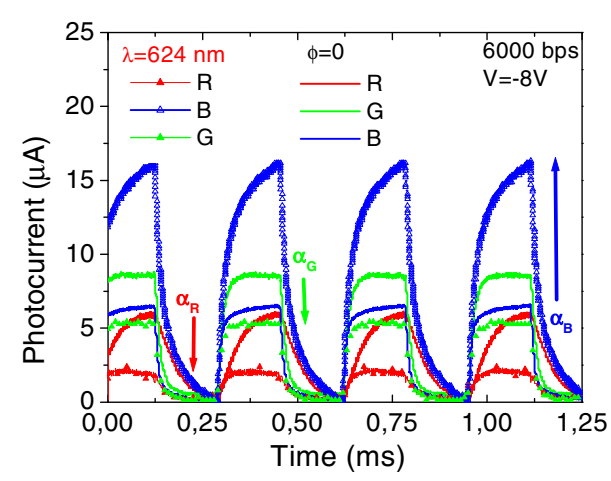

(a)

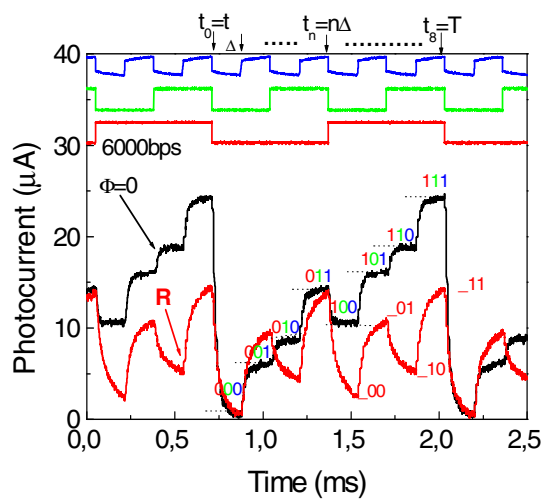

(b)

Fig. 3. a) Input red (R) green $(\mathrm{G})$ and blue (B) channels and b) Output waveform signal at $8 \mathrm{~V}$; without $(\varphi \mathrm{L}=0)$ and with $(\mathrm{R})$ optical bias

Results show that under steady state irradiation the red background enhances the light-to-dark sensitivity in the short wavelength range and quenches it in the long wavelength range. Since, under red illumination, the electrical field increases locally at least absorbing cell [3], the front diode (self optical amplification) the collection due to the carriers generated by the blue channel (absorbed in the front diode) is enhanced (Fig. 3a). The channel gain, defined as the ratio between the channel signal with and without steady state optical bias becomes higher than one $\left(\alpha_{B}>>1\right.$, see arrows). In the back diode the electric field is reduced (self optical quench) so, the collection of the carrier generated by the red channel is reduced. Here, the gain becomes lower than one $\left(\alpha_{R}<<1\right)$. The green channel is mostly absorbed across the back diode due to its higher thickness, so its gain is also lower than one $\left(\alpha_{\mathrm{G}}<1\right)$ but higher than $\alpha_{R}$ due to the front carrier collection.

When the polychromatic combination of the different pulsed channels impinges on the device, under red steady state additional optical bias, the output signal has a strong nonlinear dependence on the light absorption profile in a mode that induces a nonlinear wavelength bias dependent gain. This gain depends on the background wavelength that controls the input signals (Fig. 3a). 
To recover the transmitted information (6000bps, 8 bit per wavelength channel) the output waveforms under red irradiation and without it were used. Both multiplexed signals, during a complete cycle $(\mathrm{T})$, were divided into eight time slots $(\Delta=133 \mu \mathrm{s})$, each one with a bandwidth of $7.5 \mathrm{KHz}$ corresponding to one bit where the independent optical signals can be ON (1) or OFF (0). In Fig. 3 all the possible combination of the three input channels are present, so, the waveform of the output without optical bias is, as in Fig. 2b, an 8-level encoding $\left(2^{3}\right)$ to which it corresponds 8 different photocurrent thresholds. Taking into account Fig. 3, under red background the red channel is strongly quenched and the blue enhanced. So, the output waveform becomes a main 4-level encoding $\left(2^{2}\right)$. Here, the higher level corresponds to both blue and green ON (_11) and the lower to the absence of both (_00). The other two intermediate levels are ascribed, the upper level to the ON state of the blue (_01) channel and the lower to the green channel ON (_10). To decode the red channel, similar time slots with red background have to be compared with the ones without it, being higher the ones with the red channel ON. Using this simple algorithm the independent red, green and blue bit sequences were decoded as: B [01010101], G [00110011] and R [00001111].

\section{Photo Capacitive Active Band Pass Filter Model}

Based on the experimental results and device configuration an optoelectronic model supported by the complete dynamical large signal Ebers-Moll model with series resistors and capacitors was developed $[4,5]$. The equivalent circuit is displayed in Fig. 4 where $\alpha_{1}$ and $\alpha_{2}$ coefficients determine how the background affects the state change. In Fig. 5 it is displayed the block diagram of the optoelectronic state model for the pi'npin device under different electrical and optical bias conditions. The input signals, $\lambda_{1}, \lambda_{2}, \lambda_{3}$ model the color channels and $\mathrm{i}(\mathrm{t})$ the output signal.

$\mathrm{R}_{1}$ and $\mathrm{R}_{2}$ are the dynamic internal and back resistances and $\alpha_{1}$ and $\alpha_{2}$ are the coefficients for the steady state irradiation. Taking into account Fig. 3, under red light background we consider $\alpha_{1}>1\left(\alpha_{B}+\alpha_{G}>1\right)$ and $\alpha_{2}<1\left(\alpha_{R}+\alpha_{G}<1\right)$. The opposite will occur under blue irradiation. Under green background both are balanced. The Kirchhoff's laws give the state-space realization of the equivalent circuit. The time periodic linearized state equations are given by:

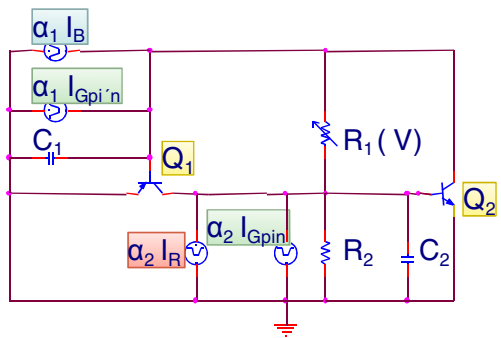

Fig. 4. ac equivalent circuit 


$$
\frac{d v_{1,2}}{d t}=\left[\begin{array}{l}
\frac{\alpha_{1}}{C_{1}} \\
\frac{\alpha_{2}}{C_{2}}
\end{array}\right] i_{1,2}(t)+\left[\begin{array}{ccc}
-\frac{1}{R_{1} C_{1}} & \frac{1}{R_{1} C_{1}} \\
\frac{1}{R_{1} C_{2}} & -\frac{1}{R_{1} C_{2}}-\frac{1}{R_{2} C_{2}}
\end{array}\right] v_{1,2}(t) i(t)=\left[\begin{array}{cc}
0 & \frac{1}{R_{2}}
\end{array}\right] v_{1,2}(t)
$$

The operation of this capacitive active circuit is based on the pi'npin device ability to simulate resistors and capacitors, resulting in integrated filters. The lack of external capacitors and inductors reduces the problems associated with those components while adding some interesting new capabilities. The use of amplifying elements $\left(\mathrm{Q}_{1}\right.$, $\left.\mathrm{Q}_{2}\right)$, with resistors $\left(\mathrm{R}_{1}, \mathrm{R}_{2}\right)$ and capacitors $\left(\mathrm{C}_{1}, \mathrm{C}_{2}\right)$ in their feedback loops, synthesize the desired filter characteristics.

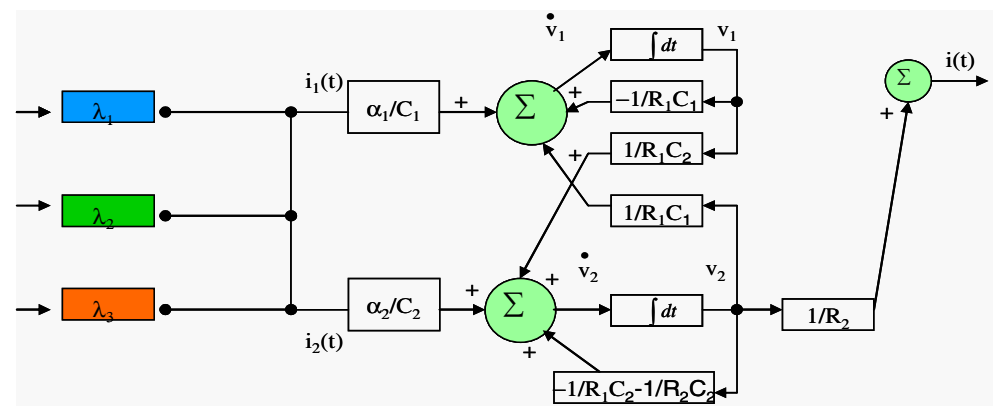

Fig. 5. Block diagram of the optoelectronic state model for a pi'n/pin device

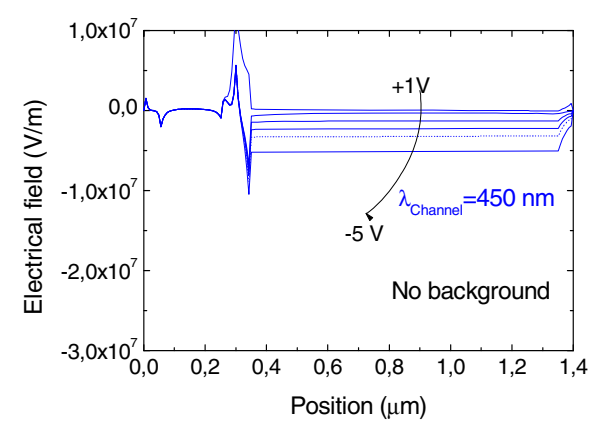

(a)

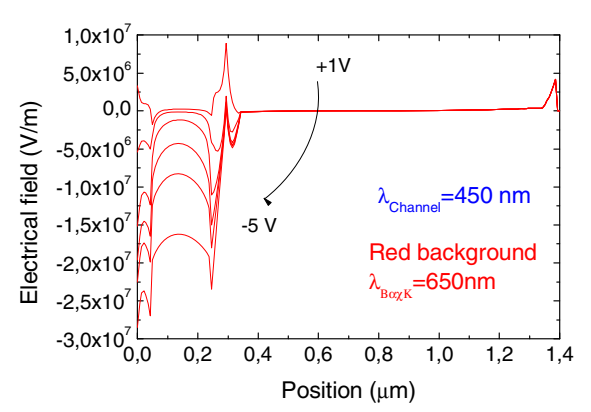

(b)

Fig. 6. Electrical Field profile using a) the blue channel and b) the blue channel and red optical bias

Taking into account equation 1 , the amplifying elements, $\alpha_{1}$ and $\alpha_{2}$, in the control matrix, can provide gain if needed or attenuate unwanted wavelengths $(<1)$ while amplifying $(>1)$ desired ones under suitable optical bias wavelengths. Under optical bias, the control matrix takes into account the enhancement or quenching of the channels (Fig. 3) due to the steady state irradiation. It will be affected by the reverse photo capacitances, $\left(\alpha_{1,2} / \mathrm{C}_{1 \cdot 2}\right)$ that determine how the system input affects the state change. 
In Fig. 6a it is displayed the electrical field profile [6], at different values of the electric bias, under the effect of the blue channel. In Fig. $6 \mathrm{~b}$ a red steady state optical bias was superimposed.

As expected as the voltage changes from positive to negative the electrical field increases in the less absorbing cell, the back diode. The addition of the red background quenches the field in the back diode where the red photons are absorbed (reverse bias effect) and increases it (reverse bias effect) in the front diode where the blue channel generates the optical carriers. So, the induced variation of the field brings on the blue collection, from which results the observed amplification of the signal (Fig. 3).

Under red background light the photogenerated charge accumulations from deep trap levels is maximized for the blue channel and correspond to a photo capacitance behavior of the internal recombination junction (considered as a measure of the density of photogenerated carriers in the space charge region) that combined with the series and parallel resistance of the diodes will result in the explicit definition of cut off wavelengths.

The values and the strategic placement of the resistors in the system matrix determine the basic shape of the output signals (output matrix). Under negative bias applied background the device has low ohmic resistance (low $\mathrm{R}_{1}$ ) since the base emitter junction of both transistors are reverse polarized and conceived as phototransistors. This results in a charging current gain proportional to the ratio between both collector currents $\left(\alpha_{1} \mathrm{C}_{2} / \alpha_{2} \mathrm{C}_{1}\right)$.

To solve the state equations the four order Runge-Kutta method was applied and MATLAB used as a programming environment. The input parameters were chosen in compliance with the experimental results. In Fig. 7a the simulated current without background $\left(\alpha_{1}=\alpha_{2}=1\right)$ under positive and negative applied voltage is displayed. In Fig. $7 \mathrm{~b}$ the simulated current under negative bias but under red background is shown. The current sources are also displayed (dash lines).

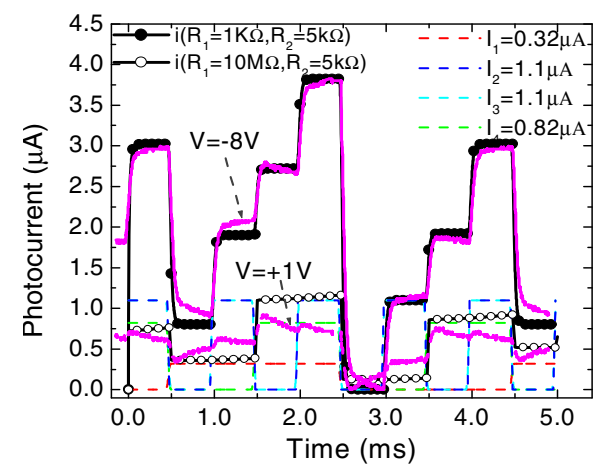

(a)

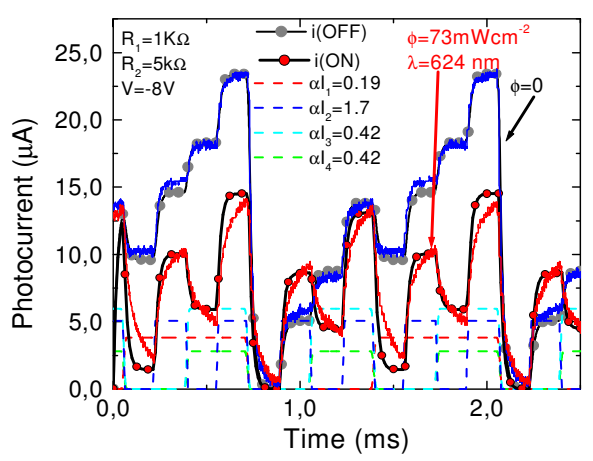

(b)

Fig. 7. Multiplexed simulated (symbols), current sources (dash lines) and experimental (solid lines): a) under negative $\left(\mathrm{R}_{1}=1 \mathrm{k} \Omega ;-8 \mathrm{~V}\right)$ and positive $\left(\mathrm{R}_{1}=10 \mathrm{M} \Omega ;+1 \mathrm{~V}\right)$ dc bias without any background; b) under negative $\left(\mathrm{R}_{1}=1 \mathrm{k} \Omega ;-8 \mathrm{~V}\right)$ and red (b) background $\left(\varphi=73 \mathrm{mWcm}^{-2}\right)$

To simulate the negative and positive electrical bias two values of $\mathrm{R}_{1}$ were used, a low one under negative bias since the internal junction is forward bias and a high value, under positive bias, due to its reverse state. For the intensity of the current 
sources the amplitude of the input channel from Fig. 2a were used. To simulate the red background, current sources intensities were multiplied by the on/off ratio between the input channels with and without optical bias (Fig. 3a). To validate the model the experimental multiplexed signals are also shown (solid lines).

A good agreement between experimental and simulated data was achieved. The device behaves like an optoelectronic controlled transmission system that stores, amplifies and transports the minority carriers generated by the current pulses, through the capacitors $\mathrm{C}_{1}$ and $\mathrm{C}_{2}$. Here, the $d c$ voltage control creates a voltage across one or both capacitors which, when superimposed with an $a c$ pulse, collectively saturates the circuit. Under red background the expected optical amplification in the low wavelength range is observed due to the effect of the active multiple-feedback filter when the back diode is light triggered.

\section{Conclusions}

$\mathrm{SiC}$ multilayer photonic structures with self optical bias amplification were analyzed. Results show that the light-activated photonic device combines the demultiplexing operation with the simultaneous photodetection and self amplification of an optical signal. The output waveform presents a nonlinear amplitude-dependent response to the wavelengths of the input channels.

A photo capacitive optical filter model was presented and supported by a numerical simulation. An algorithm to decode the multiplexed signals was established.

Acknowledgements. This work was supported by FCT (CTS multi annual funding) through the PIDDAC Program funds and PTDC/EEA-ELC/111854/2009.

\section{References}

1. Vieira, M.A., Louro, P., Vieira, M., Fantoni, A., Garção, A.S.: Light-activated amplification in Si-C tandem devices: A capacitive active filter model. IEEE Sensors Journal (2011) (in print)

2. Vieira, M., Fantoni, A., Fernandes, M., Louro, P., Lavareda, G., Carvalho, C.N.: Pinpín and pinpii'n multilayer devices with voltage controlled optical readout. Journal of Nanoscience and Nanotechnology 9(7), 4022-4027 (2009)

3. Vieira, M., Fantoni, A., Louro, P., Fernandes, M., Schwarz, R., Lavareda, G., Carvalho, C.N.: Vacuum 82(12), 1512-1516 (2008)

4. Vieira, M.A., Vieira, M., Fernandes, M., Fantoni, A., Louro, P., Barata, M.: Voltage Controlled Amorphous Si/SiC Phototransistors and Photodiodes as Wavelength Selective Devices: Theoretical and Electrical Approaches. In: Amorphous and Polycrystalline Thin-Film Silicon Science and Technology — 2009, MRS Proceedings, vol. 1153, A08-03 (2009)

5. Vieira, M.A., Vieira, M., Costa, J., Louro, P., Fernandes, M., Fantoni, A.: Double pin Photodiodes with two Optical Gate Connections for Light Triggering: A capacitive twophototransistor model. Sensors \& Transducers Journal 9, 96-120 (2010)

6. Vieira, M.A., Louro, P., Vieira, M., Fantoni, A., Garção, A.S.: Light-activated amplification in Si-C tandem devices: A capacitive active filter model. IEEE Sensors Journal (2011) (in print) 